\title{
Equilibrium Analysis of Capacity Allocation with Demand Competition
}

\author{
Zhixin Liu \\ Department of Management Studies, College of Business, University of Michigan-Dearborn, Dearborn, \\ Michigan 48126-2638 zhixin@umd.umich.edu
}

Received 20 April 2010; revised 16 February 2012; accepted 16 February 2012

DOI 10.1002/nav.21486

Published online 10 March 2012 in Wiley Online Library (wileyonlinelibrary.com).

\begin{abstract}
This article examines the capacity allocation decisions in a supply chain in which a supplier sells a common product to two retailers at a fixed wholesale price. The retailers order the supplier's product subject to an allocation mechanism preannounced by the supplier, and compete for the customer demand. We perform an equilibrium analysis of the retailers' ordering decisions under uniform and individually responsive allocations. Uniform allocation guarantees equilibrium orders, but is not necessarily truth inducing in the presence of demand competition. Further, we find that (1) neither the supplier nor either one of the retailers sees its profits necessarily increasing with the supplier's capacity, and the supplier may sell more with a lower capacity level, and (2) capacity allocation may not only affect the supply chain members' profits but also change the supply chain structure by driving a retailer out of the market. This article provides managerial insights on the capacity and ordering decisions for the supplier, the retailers, and the supply chain. (C) 2012 Wiley Periodicals, Inc. Naval Research Logistics 59: 254-265, 2012
\end{abstract}

Keywords: capacity allocation; noncooperative game; equilibrium analysis

\section{INTRODUCTION}

Capacity allocation frequently occurs in supply chains when multiple retailers order a product from a common supplier. Capacity is subject to allocation when the supplier's capacity is unable to meet retailer demand. Further, the retailers may compete for customer demand for the product. This work is specifically motivated by a capacity allocation problem in the automobile industry. Automobile manufacturers often sell the same model vehicles to multiple dealers in the same geographic region at a fixed wholesale price. The dealers are allowed to determine their own retail prices, which are typically lower than the manufacturer's suggested retail price. In this case, the dealers compete for both the manufacturer's vehicle allocation and their customer demand. Similar capacity allocation also occurs in the sales of office and home supplies, electronics products, textile and apparel products, and so forth.

This article examines the capacity allocation decisions in a supply chain, in which a supplier sells a common product to two retailers. The supplier charges a fixed wholesale price and allocates his capacity based on the order sizes of the two retailers, using a preannounced capacity allocation mechanism. The retailers order from the supplier and compete for

Correspondence to: Zhixin Liu (zhixin@umd.umich.edu) customer demand. We perform an equilibrium analysis of the retailers' ordering decisions under two types of allocation rules. The first allocation rule is uniform allocation, where the retailer with the smaller order size receives the minimum of her order size and half the supplier's capacity, whereas the other retailer receives the minimum of her order size and the remainder of the capacity. See [22,29], for how uniform allocation applies to the case with more than two retailers. We compare uniform allocation with the class of individually responsive (IR) allocations, where a retailer receives allocations that strictly increase with her order size, given that the competitor's order remains unchanged. A broad class of allocation rules are IR, such as proportional and linear allocation rules. Proportional allocation allocates capacity in proportion to order size. Linear allocation awards each retailer her order size minus a common deduction, given that this difference is non-negative, where the deduction is computed using the available capacity and the order sizes [4]. When retailers face independent demand, uniform allocation has several desirable properties. First, uniform allocation guarantees equilibrium orders. Second, uniform allocation is truth inducing in that, independent of the supplier's capacity, each retailer ordering her individually optimal order size forms an equilibrium. Third, compared with IR allocation, uniform allocation often results in higher profits for the supply chain when the wholesale price is low, and for both the supply chain 
and the retailers when no equilibrium orders exist under IR allocation [5].

We consider demand competition where the retail price from each retailer is a linear function of the quantities of the product sold by both retailers and have the following findings. First, no equilibrium orders may exist under IR allocation; uniform allocation guarantees equilibrium orders but is not necessarily truth inducing. Second, IR allocation favors the supplier in that for the same capacity size, the supplier can expect to sell at least an equal amount of capacity using IR allocation compared with using uniform allocation. Third, uniform allocation coordinates the supply chain better than IR allocation in the worst case and is most valuable in coordinating the supply chain when the difference between the retail prices of the two retailers is large, the wholesale price is low, or the supplier's capacity cost is low. Fourth, neither the supplier nor either one of the retailers sees its profits necessarily increasing with the supplier's capacity, and the supplier may sell more with a lower capacity level. Finally, capacity allocation may not only affect the supply chain members' profits but also change the supply chain structure by driving a retailer out of the market.

Insufficient capacity may originate from either the demand or the supply side. On the demand side, certain industries naturally experience volatile demand, such as those with a strong fashion component or occasional demand surges [10], and industries characterized by rapid technological development [19]. The supply side may generate capacity allocation issues in two respects. First, the supplier may not be able to adjust capacity to meet changing demands, as in capital-intensive industries [8]. Second, the supplier may strategically choose a low capacity to stimulate competition between the retailers, and thus gain more profit for himself [4], as discussed in Section 3.

Although another way of mediating demand is through pricing, price changes often lead to competitive moves by other suppliers selling similar products; thus, suppliers are often reluctant to modify the wholesale price. Capacity allocation is, therefore, an important choice for suppliers facing a demand surge. Specifically, a capacity allocation mechanism allocates capacity from suppliers to retailers based on a preannounced allocation rule, supply capacity, and order requirements. Typically, suppliers choose a capacity allocation mechanism that optimizes their own profit. However, a misallocation of capacity among retailers can result in excess capacity in parts of the supply chain and deficiencies in others, which can affect the profits of all supply chain members.

There is an extensive body of work on capacity allocation. Hall and Liu [12] provide a comprehensive review. It is well known that allocating capacity in proportion to order size induces over-ordering and contributes to the bullwhip effect [23]. Cachon and Lariviere [3] describe a turn-and-earn mechanism that allocates capacity based on past sales and demonstrate that this mechanism can benefit the supplier at the expense of the retailers and the overall supply chain. When capacity is mildly tight, the supplier can change his wholesale price and capacity level to compensate for retailer and overall supply chain profits. Cachon and Lariviere $[4,5]$ analyze incentives, equilibrium ordering decisions, and supply chain performance under a variety of capacity allocation mechanisms, and demonstrate how several allocation schemes are vulnerable to false information and show that truth inducing schemes help allocate capacity among retailers but can distort supplier choice of total capacity. Fang and Whinston [9] design an option contract for a supply chain where, from the supplier's perspective, each retailer's marginal utility for the capacity is either high or low. This design achieves the same expected profit as when the supplier knows the number of retailers of each type before investing in capacity. Ganesh et al. [11] develop a congestion pricing mechanism for allocating bandwidth in communication networks, and show that if all users predict a particular unit price and select their transmission rates accordingly, then the resulted price coincides with the prediction. Hall and Liu [13] examine integrated capacity allocation and production scheduling decisions and quantify the benefits of incorporating scheduling decisions into capacity allocation. In addition, market-based allocation mechanisms have been widely studied [14, 21, 28, 31]. Capacity allocation models with specific applications have also been discussed, such as in semiconductor manufacturing [20,24] and network communications [25]. The most closely related works to this study are those of $[4,5]$. The main difference is that we consider demand competition between retailers as motivated by practical applications.

All the studies mentioned above assume that each retailer is monopolistic in her own market and that the demands of the retailers are independent of each other. Marketing channels where a supplier sells a common product to multiple retailers with horizontal competition have been extensively studied. We study a specific supply chain structure where a supplier sells through two retailers competing for customer demand. Ingene and Parry [15] investigate channel coordination in such a setting, where the two retailers are equally treated. In the same setting, Padmanabhan and Png [27] examine how to achieve channel coordination via supplier return policies, and Ingene and Parry [16] derive an optimal wholesale pricing strategy for the supplier by simultaneously determining the wholesale price and a fixed charge. Ingene and Parry [17] recognize conditions under which a channelcoordinating wholesale price strategy generates more profit for the supplier than a sophisticated Stackelberg two-part tariff. Xiao and Qi [33] study channel coordination when the supplier's production is disrupted. Cai [6] compares this supply chain structure with others using Pareto zone concepts in the presence of channel coordination. 
In addition, Iyer [18] investigates channel coordination where two retailers compete on both price and service level. Bernstein and Federgruen [1] study the pricing and inventory replenishment decisions in a two-echelon system where a distributor sells to multiple retailers for each of which the demand rate depends on the prices of all retailers. Further, Bernstein and Federgruen [2] examine a stochastic general equilibrium inventory model where a distributor sells to multiple retailers who hold inventory to meet uncertain demand and compete on both price and service level. Raju and Zhang [26] consider channel coordination in the presence of a dominant retailer among multiple competing retailers. All these works assume that the supplier has sufficient capacity to meet all the retailer demand. Tsay and Agrawal [30] provide a comprehensive review of conflict and coordination issues and their resolutions in multichannel distribution systems.

The remainder of this article is organized as follows. Section 2 formally defines the capacity allocation problem and specifies how game issues arise. Section 3 analyzes the capacity allocation game in detail. Finally, Section 4 concludes the work.

\section{MODEL SETUP}

Suppose that two retailers, denoted 1 and 2, order a single product from a common supplier. The two retailers are in the same market and thus compete for customer demand. We consider Cournot competition, that is, the two retailers compete on the quantity of the product they sell. We assume that the two retailers face linear price functions and incur an ordering cost proportional to the size of capacity received. Specifically, each retailer $i$ incurs a cost $w$ in obtaining one unit of the product, sells $q_{i}$ units of the product at a unit price $p_{i}$, and generates a profit $\pi_{i}\left(q_{1}, q_{2}\right)$ when the two retailers sell $q_{1}$ and $q_{2}$ units of the product, respectively. We have

$$
p_{i}=z_{i}-\left(q_{1}+q_{2}\right)
$$

and

$$
\begin{aligned}
\pi_{i}\left(q_{1}, q_{2}\right)= & \left(p_{i}-w\right) q_{i}=\left(z_{i}-\left(q_{1}+q_{2}\right)-w\right) q_{i}, \\
& \text { for } i=1,2,
\end{aligned}
$$

where for the same quantity sold, a retailer with a larger $z_{i}$ charges a higher price for the product. Without loss of generality, we assume $z_{1} \geq z_{2}$. Borrowing the notations used by Cachon and Lariviere [5], we name retailer 1 a high-type retailer, and retailer 2 a low-type retailer. To have a positive order size from both retailers, we assume $z_{2}>w$.

\section{ASSUMPTION $1: z_{1} \geq z_{2}>w$}

Our analysis focuses on a pure-strategy Nash equilibrium. In a pair of equilibrium sales quantities, denoted by
$\mathbf{Q}^{*}=\left(q_{1}^{*}, q_{2}^{*}\right)$, quantity $q_{i}^{*}$ is the best response, given $q_{j}^{*}$, for $i, j=1,2$ and $i \neq j$. The first-order condition of profit functions (2) defines the unique equilibrium sales quantities, $\left(q_{1}^{*}, q_{2}^{*}\right)$, where $q_{1}^{*}=\left(2 z_{1}-z_{2}-w\right) / 3$ and $q_{2}^{*}=\left(2 z_{2}-z_{1}-w\right) / 3$, given that $q_{2}^{*}>0$. To guarantee $q_{2}^{*}>0$, we make the following assumption.

\section{ASSUMPTION 2: $z_{2}>\left(z_{1}+w\right) / 2$.}

Assumption 2 prevents retailer 1 from becoming a monopoly when capacity is unlimited. Further, we assume that both retailers sell the entirety of their filled orders, that is, sales quantity $q_{i}$ is the filled order size of retailer $i$. If stock withholding is considered, then given retailer $j$ 's sales quantity $q_{j}$ and retailer $i$ 's filled order size $x_{i}$, retailer $i$ 's maximum profit is

$$
\begin{aligned}
& \max _{0 \leq q_{i} \leq x_{i}}\left\{\left(z_{i}-\left(q_{i}+q_{j}\right)-w\right) q_{i}-w\left(x_{i}-q_{i}\right)\right\} \\
= & \begin{cases}\left(z_{i}-\left(x_{i}+q_{j}\right)-w\right) x_{i}, & \text { if } x_{i} \leq\left(z_{i}-q_{j}\right) / 2, \\
\left(\left(z_{i}-q_{j}\right) / 2\right)^{2}-w x_{i}, & \text { if } x_{i}>\left(z_{i}-q_{j}\right) / 2,\end{cases}
\end{aligned}
$$

where $q_{i}$ and $x_{i}-q_{i}$ are retailer $i$ 's sales and withholding quantities, respectively. Retailer $i$ should withhold stock when her filled order size is greater than $\left(z_{i}-q_{j}\right) / 2$. However, each retailer's profit function is a strictly concave function of allocated capacity, whether or not stock is being withheld. Therefore, managerial insights obtained without considering stock withholding do not lose qualitative generality. In practice, the retailers may not be able to withhold stock. For example, many automobile manufacturers have websites displaying a detailed inventory of their dealers. In addition, the assumption is consistent with the capacity allocation analysis of [5], and thus, we can compare their results with ours.

\section{ASSUMPTION 3: Retailers do not withhold stock.}

Now, we consider the supplier's capacity allocation. Suppose the supplier has $K$ units of capacity, with cost normalized to zero. Our equilibrium analysis does not lose generality, as the retailers do not need to know the supplier's capacity cost. We consider capacity allocations that are based on the order sizes of the two retailers. Let $\mathbf{R}=\left(r_{1}, r_{2}\right)$ denote a pair of order sizes where retailer $i$ orders $r_{i}$ units of capacity. Let $\mathbf{X}=\left(x_{1}, x_{2}\right)$ denote a pair of allocation sizes where retailer $i$ receives $x_{i}$ units of capacity. An allocation rule is a function that defines $\mathbf{X}$ based on $K$ and $\mathbf{R}$. For example, under the proportional allocation rule, we have

$$
x_{i}=\min \left\{r_{i}, r_{i} K /\left(r_{1}+r_{2}\right)\right\}, \quad i=1,2 .
$$


Under the linear allocation rule, we have

$x_{i}= \begin{cases}r_{i}, & \text { if } r_{i}+r_{j} \leq K, \\ \left(r_{i}-r_{j}+K\right) / 2, & \text { if } r_{i}+r_{j}>K,\left|r_{i}-r_{j}\right| \leq K, \\ K, & \text { if } r_{i}+r_{j}>K, r_{i}-r_{j}>K, \\ 0, & \text { if } r_{i}+r_{j}>K, r_{i}-r_{j}<-K,\end{cases}$

where $i, j=1,2$ and $j \neq i$.

Under the uniform allocation rule, we have

$$
x_{i}= \begin{cases}r_{i}, & \text { if } r_{i}+r_{j} \leq K, \\ \min \left\{r_{i}, K / 2\right\}, & \text { if } r_{i}+r_{j}>K, r_{i} \leq r_{j}, \\ \max \left\{K-r_{j}, K / 2\right\}, & \text { if } r_{i}+r_{j}>K, r_{i}>r_{j},\end{cases}
$$

where $i, j=1,2$ and $j \neq i$.

According to the definitions of [4], an allocation rule is efficient, if $r_{1}+r_{2} \leq K$ implies $x_{i}=r_{i}$ and $r_{1}+r_{2}>K$ implies $x_{1}+x_{2}=K$. Obviously, proportional, linear, and uniform allocations are all efficient. Proportional and linear allocations are also IR, but uniform allocation is not. Our analysis applies to any IR allocation, rather than specifically to proportional or linear allocation.

If both retailers' orders are guaranteed to be filled by the supplier, then obviously the equilibrium order sizes are the same as the equilibrium sales quantities $\left(q_{1}^{*}, q_{2}^{*}\right)$. Let $K^{*}=q_{1}^{*}+q_{2}^{*}=\left(z_{1}+z_{2}-2 w\right) / 3$. With demand competition, we designate an allocation rule as truth inducing if the equilibrium sales quantities are also the equilibrium order sizes, independent of the supplier's capacity size $K$.

\section{CAPACITY ALLOCATION GAME}

This section analyzes the capacity allocation game, specifically how uniform and IR allocations affect the profits of the supplier, the retailers, and the supply chain. Sections 3.1 and 3.2 conduct equilibrium analysis of ordering decisions under uniform and IR allocations, respectively. Section $3.3 \mathrm{com}$ pares uniform and IR allocations from the perspectives of the supplier and retailers, respectively. Section 3.4 discusses the inefficiency of capacity allocation relative to a centralized supply chain. Section 3.5 conducts a computational study to gain insights on how the profits of supply chain members are affected by various factors. Finally, Section 3.6 compares capacity allocations with and without demand competition.

\subsection{Equilibrium Analysis: Uniform Allocation}

Under uniform allocation, a retailer can always obtain half of the supplier's capacity $K$ by ordering $K / 2$. In this case, when the supplier's capacity is bounded, a retailer may be able to increase her profit by deviating from the order pair $\left(q_{1}^{*}, q_{2}^{*}\right)$ to gain half of the supplier's capacity. Therefore, our equilibrium analysis considers situations where each retailer receives half the supplier's capacity.

For the capacity allocation problem defined in Section 2, let $f_{2}=-8 z_{1}^{2}+32 z_{1} z_{2}-23 z_{2}^{2}-16 z_{1} w+14 z_{2} w+w^{2}$, $K_{2}^{-}=\left(z_{2}-w\right) / 2-\sqrt{f_{2}} / 6$, and $K_{2}^{+}=\left(z_{2}-w\right) / 2+\sqrt{f_{2}} / 6$, where $K_{2}^{-}$and $K_{2}^{+}$are solutions of $\left(z_{2}-w-k\right) k / 2=$ $\left(z_{2}-w-K^{*}\right) q_{2}^{*}$ with variable $k$. It can be verified that $f_{2}>0$ under Assumptions 1 and 2. By definition, retailer 2 gains the same profit from allocation pairs $\left(q_{1}^{*}, q_{2}^{*}\right),\left(K_{2}^{-} / 2, K_{2}^{-} / 2\right)$, and $\left(K_{2}^{+} / 2, K_{2}^{+} / 2\right)$, and gains more profit from $(K / 2, K / 2)$ than from $\left(q_{1}^{*}, q_{2}^{*}\right)$ when $K_{2}^{-}<K<K_{2}^{+}$. Next, we compare $K^{*}, K_{2}^{-}, K_{2}^{+}, 2 q_{1}^{*}$, and $z_{2}-w$. Note that by profit functions (2), if the total size of allocated capacity is no less than $z_{2}-w$, retailer 2 gains no profit.

LEMMA 1: $K_{2}^{-} \leq K^{*} \leq K_{2}^{+} \leq \min \left\{2 q_{1}^{*}, z_{2}-w\right\}$.

PROOF: First, we show $K_{2}^{-} \leq K^{*} \leq K_{2}^{+}$. By the concavity of $\pi_{2}(k / 2, k / 2)$, it suffices to show

$$
\pi_{2}\left(q_{1}^{*}, q_{2}^{*}\right) \leq \pi_{2}\left(K^{*} / 2, K^{*} / 2\right),
$$

which holds following the definition of $q_{1}^{*}, q_{2}^{*}$, and $K^{*}$, and that $q_{2}^{*} \leq K^{*} / 2$.

Second, we show $K_{2}^{+} \leq 2 q_{1}^{*}$. By the concavity of $\pi_{2}(k / 2, k / 2)$, it suffices to show

$$
\pi_{2}\left(q_{1}^{*}, q_{2}^{*}\right) \geq \pi_{2}\left(q_{1}^{*}, q_{1}^{*}\right),
$$

which is $\left(z_{1}-z_{2}\right)^{2} \geq 0$, which holds.

Third, we show $K_{2}^{+} \leq z_{2}-w$. By profit functions (2), $\pi_{2}\left(\left(z_{2}-w\right) / 2,\left(z_{2}-w\right) / 2\right)=0$, and thus the inequality holds.

Lemma 1 indicates that there exists a capacity size $K$, $K^{*}<K<K_{2}^{+}$, under which, given retailer 1's order size $q_{1}^{*}$, retailer 2 can increase her order size from $q_{2}^{*}$ to no less than $K / 2$, and thus gain more profit from the resulting allocation pair $(K / 2, K / 2)$ than from $\left(q_{1}^{*}, q_{2}^{*}\right)$. Therefore, even when $K>K^{*}$, the order pair $\left(q_{1}^{*}, q_{2}^{*}\right)$ may not be in equilibrium.

THEOREM 1: Under uniform allocation and with price and profit functions defined by Eqs (1) and (2), respectively, equilibrium order pairs and their resulting allocation pairs are provided in Table 1. Furthermore, under each of the two conditions of $K$, no other allocation pair can result from an equilibrium order pair.

Table 1. Equilibrium under uniform allocation.

\begin{tabular}{lcc}
\hline Capacity $K$ & Equilibrium & Allocation \\
\hline$K<K_{2}^{+}$ & $(K, K)$ & $(K / 2, K / 2)$ \\
$K>K_{2}^{+}$ & $\left(q_{1}^{*}, q_{2}^{*}\right)$ & $\left(q_{1}^{*}, q_{2}^{*}\right)$ \\
\hline
\end{tabular}

Naval Research Logistics DOI 10.1002/nav 
PROOF: Before proving the theorem, we need to define and compare several values. Let $f_{1}=-23 z_{1}^{2}+32 z_{1} z_{2}-8 z_{2}^{2}+$ $14 z_{1} w-16 z_{2} w+w^{2}$ and let $K_{1}^{+}=\left(z_{1}-w\right) / 2+\sqrt{f_{1}} / 6$, given $f_{1} \geq 0$, which is the largest solution, if it exists, to $\left(z_{1}-w-k\right) k / 2=\left(z_{1}-w-K^{*}\right) q_{1}^{*}$ with variable $k$. By definition, retailer 1 obtains the same profit from allocation pairs $\left(q_{1}^{*}, q_{2}^{*}\right)$ and $\left(K_{1}^{+} / 2, K_{1}^{+} / 2\right)$. Next, we show that

$$
K_{1}^{+} \leq K_{2}^{+} .
$$

A necessary condition for $K_{1}^{+}$to exist is $f_{1} \geq 0$. We show that $f_{1} \geq 0$ requires $z_{1}<\left(5 z_{2}-w\right) / 4$. As $\partial f_{1} / \partial z_{1}=-46 z_{1}+32 z_{2}+14 w<0, f_{1}$ decreases with $z_{1}$. Plugging $z_{1}=\left(5 z_{2}-w\right) / 4$ into $f_{1}$, we obtain $f_{1}=-63\left(z_{2}-w\right)^{2} / 16<0$. Therefore, it is necessary to have $z_{1}<\left(5 z_{2}-w\right) / 4$ for $K_{1}^{+}$to exist. Next,

$$
K_{1}^{+}-K_{2}^{+}=\left(3 z_{1}-3 z_{2}+\sqrt{f_{1}}-\sqrt{f_{2}}\right) / 6,
$$

and thus, it suffices to show

$$
\left(3 z_{1}-3 z_{2}\right)^{2} \leq\left(\sqrt{f_{1}}-\sqrt{f_{2}}\right)^{2},
$$

which is

$$
\left(4 z_{1}-5 z_{2}+w\right)\left(-5 z_{1}+4 z_{2}+w\right) \geq \sqrt{f_{1} f_{2}},
$$

where the left-hand side is non-negative given Assumption 1 and $z_{1}<\left(5 z_{2}-w\right) / 4$. Therefore, it suffices to show

$$
\left(4 z_{1}-5 z_{2}+w\right)^{2}\left(-5 z_{1}+4 z_{2}+w\right)^{2} \geq f_{1} f_{2},
$$

which is

$$
216\left(z_{1}-z_{2}\right)^{2}\left(\left(z_{1}-z_{2}\right)^{2}+\left(z_{1}-w\right)\left(z_{2}-w\right)\right) \geq 0,
$$

which holds under Assumption 1.

We now prove the theorem. First, when $K<K_{2}^{+}$, the order pair $(K, K)$ results in the allocation pair $(K / 2, K / 2)$. By the definition of uniform allocation, changing order size to any value no less than $K / 2$ will not affect a retailer's received allocation. If a retailer reduces her order size to less than $K / 2$, then she will receive the quantity she orders. In this case, as the total size of the allocated capacity will remain $K \leq z_{2}-w$, the retailer's profit will decrease given profit functions (2). Therefore, the order pair $(K, K)$ is in equilibrium.

Now, we show that no other allocation pair can result from an equilibrium order pair. When the total size of the allocated capacity is less than $K$, by the concavity of profit functions (2), the only possible equilibrium order pair is $\left(q_{1}^{*}, q_{2}^{*}\right)$, given that $K>K^{*}$. However, by Lemma 1 , we have $K<2 q_{1}^{*}$, and thus, retailer 2 can gain more profit from allocation pair $(K / 2, K / 2)$ by increasing her order size to be no less than $K / 2$. Therefore, the order pair $\left(q_{1}^{*}, q_{2}^{*}\right)$ is not in equilibrium. When the total size of the allocated capacity is equal to $K$, clearly no other allocation pair can result from an equilibrium order pair, since whichever retailer receiving allocation in a size less than $K / 2$ can increase her order size to gain more allocation and thus more profit. Therefore, $(K / 2, K / 2)$ is the unique allocation pair resulting from an equilibrium order pair under uniform allocation when $K<K_{2}^{+}$.

Second, when $K>K_{2}^{+}$, clearly no retailer can gain more profit by reducing her order size from $\left(q_{1}^{*}, q_{2}^{*}\right)$. In addition, as $q_{1}^{*} \geq q_{2}^{*}$, when retailer 1 increases her order size, the received allocation of retailer 2 will not change, and thus, ordering $q_{1}^{*}$ is retailer 1's best response to retailer 2's order size $q_{2}^{*}$. Now, consider the case where retailer 2 increases her order size to $q>q_{2}^{*}$. If $q_{1}^{*}+q \leq K$, then, by the definition of $\left(q_{1}^{*}, q_{2}^{*}\right)$, retailer 2 cannot gain more profit. Hence, we assume $q_{1}^{*}+q>K$. If $K \geq z_{2}-w$, then retailer 2 will gain no profit from the order pair $\left(q_{1}^{*}, q\right)$, so, we need consider only the case where $K<z_{2}-w$.

1. If $q_{1}^{*} \leq q$, then the allocation pair is either $(K / 2, K / 2)$ or $\left(q_{1}^{*}, K-q_{1}^{*}\right)$, neither of which offers retailer 2 more profit than the order pair $\left(q_{1}^{*}, q_{2}^{*}\right)$, given $K>K_{2}^{+}$and the definition of $\left(q_{1}^{*}, q_{2}^{*}\right)$.

2. If $q_{1}^{*}>q$, then retailer 2's profit is maximized when the allocation is $(K / 2, K / 2)$, which is no more than that from $\left(q_{1}^{*}, q_{2}^{*}\right)$ given $K>K_{2}^{+}$.

Therefore, the order pair $\left(q_{1}^{*}, q_{2}^{*}\right)$ is in equilibrium under uniform allocation when $K>K_{2}^{+}$.

We now show that no other allocation pair can result from an equilibrium order pair, by showing that no other order pair is in equilibrium when $K>K_{2}^{+}$. If the total size of the allocated capacity is less than $K$, then the uniqueness of the equilibrium order pair $\left(q_{1}^{*}, q_{2}^{*}\right)$ follows the concavity of profit functions (2). We assume the total size of the allocated capacity equals $K$. If $K<z_{2}-w$, then the only other possible allocation resulting from an equilibrium order pair is $(K / 2, K / 2)$, otherwise, whichever retailer receiving allocation in a size less than $K / 2$ can increase her order size to gain more allocation and thus greater profit. However, under the condition $K>K_{2}^{+}$, we have $\pi_{2}\left(q_{1}^{*}, q_{2}^{*}\right)>$ $\pi_{2}(K / 2, K / 2)$ (Lemma 1) and $K>K_{1}^{+}$(inequality (4)), and thus $\pi_{1}\left(q_{1}^{*}, q_{2}^{*}\right)>\pi_{1}(K / 2, K / 2)$. Thus, any order pair resulting in the allocation pair $(K / 2, K / 2)$ is dominated by the order pair $\left(q_{1}^{*}, q_{2}^{*}\right)$. Therefore, the unique allocation pair resulting from an equilibrium order pair is $\left(q_{1}^{*}, q_{2}^{*}\right)$.

Note that when $K<K_{2}^{+}$, there exist other equilibrium order pairs under uniform allocation. Specifically, any order pair $\left(q_{1}, q_{2}\right)$ is in equilibrium when

1. $q_{1} \geq K / 2$,

2. $q_{2} \geq K / 2$, 
3. $\max _{0 \leq q \leq K-q_{2}}\left\{\pi_{1}\left(q, q_{2}\right)\right\} \leq \pi_{1}(K / 2, K / 2)$,

4. $\max _{0 \leq q \leq K-q_{1}}\left\{\pi_{2}\left(q_{1}, q\right)\right\} \leq \pi_{2}(K / 2, K / 2)$.

However, these order pairs all lead to the same allocation pair $(K / 2, K / 2)$, so their existence does not affect the allocation of capacity.

Interestingly, Lemma 1 and Theorem 1 show that, for a pair of equilibrium orders from the two retailers, with limited capacity, the supplier can sell more units of capacity than in the case with unlimited capacity. Theorem 1 also shows that with demand competition, uniform allocation guarantees the existence of an equilibrium order pair but is not necessarily truth inducing.

\subsection{Equilibrium Analysis: IR Allocation}

Under IR allocation, a retailer can obtain the supplier's entire capacity when her order size goes to infinity, holding the order size of the other retailer constant. In this case, with a bounded supplier's capacity, a retailer may be able to gain more profit by deviating from the order pair $\left(q_{1}^{*}, q_{2}^{*}\right)$ to gain the supplier's entire capacity. Therefore, our equilibrium analysis considers situations where a retailer gains the entire capacity.

For the capacity allocation problem defined in Section 2 , let $g_{1}=\left(7 z_{1}-2 z_{2}-5 w\right)\left(-z_{1}+2 z_{2}-w\right), L_{1}^{-}=$ $\left(z_{1}-w\right) / 2-\sqrt{g_{1}} / 6$, and $L_{1}^{+}=\left(z_{1}-w\right) / 2+\sqrt{g_{1}} / 6$, where $L_{1}^{-}$and $L_{1}^{+}$are solutions to $\left(z_{1}-w-k\right) k=\left(z_{1}-w-K^{*}\right) q_{1}^{*}$ with variable $k$. Note that $g_{1}>0$ under Assumptions 1 and 2 . By definition, retailer 1 obtains the same profit from allocation pairs $\left(q_{1}^{*}, q_{2}^{*}\right),\left(L_{1}^{-}, 0\right)$, and $\left(L_{1}^{+}, 0\right)$. Next, we compare $K^{*}, L_{1}^{-}, L_{1}^{+}$, and $z_{2}-w$.

LEMMA 2: 1) $L_{1}^{-} \leq K^{*} \leq L_{1}^{+}$;2) $L_{1}^{+} \leq z_{2}-w$ when $4 z_{1}-5 z_{2}+w \leq 0$ and $L_{1}^{+}>z_{2}-w$ when $4 z_{1}-5 z_{2}+w>0$.

PROOF: First, we show $L_{1}^{-} \leq K^{*} \leq L_{1}^{+}$. By the concavity of $\pi_{1}(k, 0)$, it suffices to show

$$
\pi_{1}\left(q_{1}^{*}, q_{2}^{*}\right) \leq \pi_{1}\left(K^{*}, 0\right)
$$

which holds under the definition of $q_{1}^{*}, q_{2}^{*}$, and $K^{*}$.

Second, we compare $L_{1}^{+}$and $z_{2}-w$. Note that $L_{1}^{+}-\left(z_{2}-\right.$ $w)=\left(\sqrt{g_{1}}-\left(6 z_{2}-3 z_{1}-3 w\right)\right) / 6$, and thus, $L_{1}^{+} \leq z_{2}-w$ is equivalent to

$$
\sqrt{g_{1}} \leq 3\left(-z_{1}+2 z_{2}-w\right)
$$

which is

$$
4\left(4 z_{1}-5 z_{2}+w\right)\left(-z_{1}+2 z_{2}-w\right) \leq 0 .
$$

Therefore, under Assumption 2, we have $L_{1}^{+} \leq z_{2}-w$ when $4 z_{1}-5 z_{2}+w \leq 0$, and $L_{1}^{+}>z_{2}-w$ when $4 z_{1}-5 z_{2}+w>0$.
Table 2. Equilibrium under IR allocation.

\begin{tabular}{lcc}
\hline Capacity $K$ & Equilibrium & Allocation \\
\hline$K<L_{1}^{+}$ & None & N/A \\
$K>L_{1}^{+}$ & $\left(q_{1}^{*}, q_{2}^{*}\right)$ & $\left(q_{1}^{*}, q_{2}^{*}\right)$ \\
\hline
\end{tabular}

Lemma 2 indicates that under IR allocation, even when the supplier's capacity $K$ is larger than $K^{*}$, the order pair $\left(q_{1}^{*}, q_{2}^{*}\right)$ may not be in equilibrium, as $\pi_{1}(K, 0)$ can be larger than $\pi_{1}\left(q_{1}^{*}, q_{2}^{*}\right)$. This finding also implies that the supplier may have more than $K^{*}$ units of capacity sold when his capacity size is between $K^{*}$ and $L_{1}^{+}$.

THEOREM 2: Under IR allocation and with price and profit functions defined by (1) and (2), respectively, equilibrium order pairs and their resulting allocation pairs are provided in Table 2. Furthermore, when $K>L_{1}^{+}$, no other equilibrium order pair exists.

PROOF: We first establish a preliminary result. Let $g_{2}=$ $\left(-2 z_{1}+7 z_{2}-5 w\right)\left(2 z_{1}-z_{2}-w\right)$ and $L_{2}^{+}=\left(z_{2}-w\right) / 2+$ $\sqrt{g_{2}} / 6$, where $L_{2}^{+}$is the largest solution to $\left(z_{2}-w-k\right) k=$ $\left(z_{2}-w-K^{*}\right) q_{2}^{*}$ with variable $k$. Note that $g_{2}>0$ under Assumptions 1 and 2. By definition, retailer 2 gains the same profit from the allocation pairs $\left(q_{1}^{*}, q_{2}^{*}\right)$ and $\left(0, L_{2}^{+}\right)$. Next, we compare $L_{1}^{+}$and $L_{2}^{+}$. We show that

$$
L_{1}^{+} \geq L_{2}^{+}
$$

Noting that $L_{1}^{+}-L_{2}^{+}=\left(z_{1}-z_{2}\right) / 2-\left(\sqrt{g_{2}}-\sqrt{g_{1}}\right) / 6$, we need to show

$$
9\left(z_{1}-z_{2}\right)^{2} \geq\left(\sqrt{g_{2}}-\sqrt{g_{1}}\right)^{2},
$$

which is

$$
2 \sqrt{g_{1} g_{2}} \geq 10\left(z_{1}-2 z_{2}+w\right)\left(-2 z_{1}+z_{2}+w\right),
$$

which is

$$
144\left(z_{1}-z_{2}\right)^{2}\left(2 z_{1}-z_{2}-w\right)\left(-z_{1}+2 z_{2}-w\right) \geq 0,
$$

which holds under Assumptions 1 and 2. Next, we prove the theorem.

First, we show that when $K<L_{1}^{+}$, no order pair is in equilibrium. First, consider the case in which the total size of the allocated capacity is less than $K$. By the concavity of profit functions (2), the only possible equilibrium order pair is $\left(q_{1}^{*}, q_{2}^{*}\right)$. However, by Lemma 2 , retailer 1 can gain more profit by increasing her order size to be sufficiently large, and thus $\left(q_{1}^{*}, q_{2}^{*}\right)$ is not in equilibrium. Second, consider the case in which the total size of the allocated capacity is equal to $K$. If $K<z_{2}-w$, then each retailer wants to order more to 
receive more units of capacity and thus more profit; hence, no equilibrium order pair exists. If $K \geq z_{2}-w$, then by profit functions (2), retailer 2 cannot gain a positive profit, and thus in an equilibrium order pair, her order size is zero; accordingly, retailer 1's optimal response is to order $\left(z_{1}-w\right) / 2$. However, this results in a total size $\left(z_{1}-w\right) / 2$ of the allocated capacity, which is less than $z_{2}-w$ under Assumption 2. Therefore, no equilibrium order pair exists when $K \geq z_{2}-w$.

Second, we show that when $K>L_{1}^{+}$, order pair $\left(q_{1}^{*}, q_{2}^{*}\right)$ is in equilibrium. As $K>L_{1}^{+} \geq K^{*}$, under IR allocation, obviously no retailer can increase her profit by reducing her order size from the order pair $\left(q_{1}^{*}, q_{2}^{*}\right)$. If a retailer wants to increase her profit by increasing order size, then this can be achieved only when the total size of the allocated capacity is $K$, by the concavity of profit functions (2). If the total size of the allocated capacity is $K$, then, by profit functions (2), a retailer's profit increases with order size. However, even when a retailer gains the entire capacity, her profit is still no more than that from order pair $\left(q_{1}^{*}, q_{2}^{*}\right)$, as indicated by Lemma 2 and inequality (5). Therefore, order pair $\left(q_{1}^{*}, q_{2}^{*}\right)$ is in equilibrium.

Now, we show that when $K>L_{1}^{+}$, no other order pair is in equilibrium. When the total size of the allocated capacity is less than $K$, the only equilibrium order pair is $\left(q_{1}^{*}, q_{2}^{*}\right)$, following the concavity of profit functions (2). If the total size of the allocated capacity is equal to $K$, then a retailer's profit increases with her order size. However, from Lemma 2 and inequality (5), even when a retailer gains the entire capacity $K$, her profit is still no more than that from order pair $\left(q_{1}^{*}, q_{2}^{*}\right)$, and thus order pair $\left(q_{1}^{*}, q_{2}^{*}\right)$ is the unique equilibrium order pair.

Intuitively, an equilibrium order pair cannot exist under IR allocation when $K<L_{1}^{+}$, because either retailer 1 can gain more profit by ordering more than $q_{1}^{*}$ to increase her allocated capacity and reduce retailer 2's allocated capacity or both retailers have incentive to gain all the capacity. By Lemma 2, it is possible to have $K^{*}<K<L_{1}^{+}<z_{2}-w$. In this case, by the proof of Theorem 2 , no equilibrium order pair exists, but when anticipating the entire capacity is allocated, each retailer has incentive to gain all the capacity, and as a result the entire capacity is truly allocated. That is, with limited capacity, the supplier may sell more units of capacity than in the case with unlimited capacity.

\subsection{Uniform Allocation Versus IR Allocation}

Consider how the supplier's capacity decision is affected by the allocation rule. Under uniform allocation, the supplier's capacity can be sold up to $K_{2}^{+}$, as shown in Table 1 . Under IR allocation, when $K<L_{1}^{+}$, no equilibrium order pair exists and we estimate the total size of the allocated capacity as follows. When $K<\min \left\{z_{2}-w, L_{1}^{+}\right\}$, both retailers have incentive to gain as much capacity as possible, and thus it is reasonable to assume that all the capacity is allocated. When $z_{2}-w<K<L_{1}^{+}$, as retailer 2 cannot gain any profit when the total size of the allocated capacity is no less than $z_{2}-w$, the total size of the allocated capacity can be expected to be $z_{2}-w$. Cachon and Lariviere [5] adopt a similar assumption when discussing the total size of the allocated capacity in the case of no equilibrium order pair under IR allocation. We next compare $K_{2}^{+}$with $L_{1}^{+}$and $z_{2}-w$.

LEMMA 3: (1) $K_{2}^{+} \leq L_{1}^{+}$; (2) $K_{2}^{+} \leq z_{2}-w$.

PROOF: First, we show that $K_{2}^{+} \leq L_{1}^{+}$. By inequality (5), we have $L_{2}^{+} \leq L_{1}^{+}$, and thus it suffices to show $K_{2}^{+} \leq L_{2}^{+}$. By definition, we have $\pi_{2}\left(K_{2}^{+} / 2, K_{2}^{+} / 2\right)=\pi_{2}\left(q_{1}^{*}, q_{2}^{*}\right)=$ $\pi_{2}\left(0, L_{2}^{+}\right)$, that is, $\left(z_{2}-w-K_{2}^{+}\right) K_{2}^{+} / 2=\left(z_{2}-w-L_{2}^{+}\right) L_{2}^{+}$, which indicates $K_{2}^{+} \leq L_{2}^{+}$.

Second, we compare $K_{2}^{+}$and $z_{2}-w$. Noting that retailer 2 gains no profit when the total size of the allocated capacity is no less than $z_{2}-w$, but obtains positive profit when the total size of the allocated capacity is $K_{2}^{+}$, we have $K_{2}^{+} \leq z_{2}-w$.

In view of Lemma 3, we have the following result.

REMARK 1: For the same capacity size, the supplier can expect to sell at least an equal amount of capacity using IR allocation compared with using uniform allocation.

Remark 1 shows that, with IR allocation, the supplier can expect to sell at least an equal amount of capacity as with uniform allocation. Consider a specific situation where $4 z_{1}-5 z_{2}+w>0$, that is, $z_{1}>\left(5 z_{2}-w\right) / 4$. Here, we designate retailer 1 as having significantly higher retail price than retailer 2. In this situation, the supplier's capacity level can be between $z_{2}-w$ and $L_{1}^{+}$, as indicated by Lemma 2 . At such a capacity level, retailer 1 can strategically order $z_{2}-w$, such that retailer 2 can gain no profit.

\subsection{Inefficiency of Decentralization}

This work considers capacity allocation in a decentralized supply chain. One can anticipate that strategic ordering and allocation decisions will result in inefficiency; that is, the supply chain profit resulting from capacity allocation is lower than that of the centralized supply chain. Unfortunately, the inefficiency of decentralization can be significant.

REMARK 2: With price and profit functions defined by (1) and (2), respectively, under uniform or IR allocation, the ratio of the supply chain profit from an equilibrium order pair to the maximum profit of the centralized supply chain approaches zero in the worst case. 


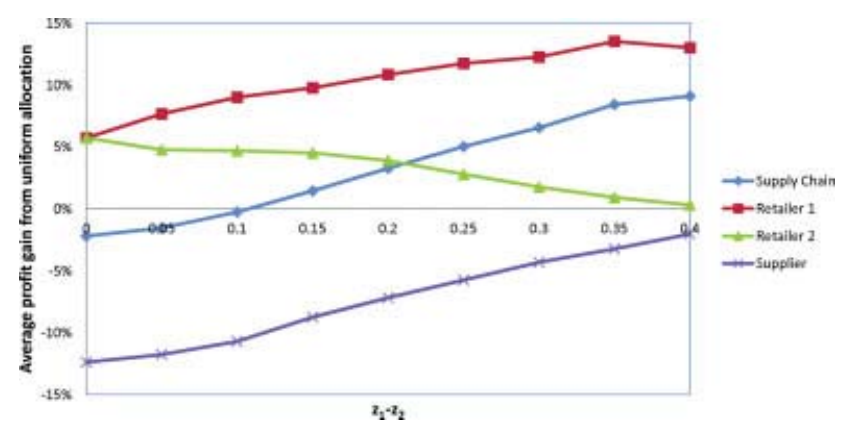

Figure 1. Profit gain as a function of $z_{1}-z_{2}$. [Color figure can be viewed in the online issue, which is available at wileyonlinelibrary. com.]

PROOF: Consider the case where the supplier has unlimited capacity. The maximum profit of the centralized supply chain is obtained when the supplier allocates capacity $z_{1} / 2$ to retailer 1 and zero to retailer 2 , such that the supply chain profit is $\pi_{S}^{*}=z_{1}^{2} / 4$. On the other hand, the equilibrium order pair $\left(q_{1}^{*}, q_{2}^{*}\right)$ results in a supply chain profit of $\pi_{S}^{L}=\left(5 z_{1}^{2}-8 z_{1} z_{2}+5 z_{2}^{2}+z_{1} w+z_{2} w-4 w^{2}\right) / 9$. Then, we have

$$
\frac{\pi_{S}^{L}}{\pi_{S}^{*}}=\frac{4\left(5 z_{1}^{2}-8 z_{1} z_{2}+5 z_{2}^{2}+z_{1} w+z_{2} w-4 w^{2}\right)}{9 z_{1}^{2}},
$$

which approaches zero when $z_{2} \rightarrow z_{1}$ and $w \rightarrow z_{2}$.

The proof of Remark 2 does not involve capacity allocation issues, as the proof uses an instance with unlimited capacity. It is more interesting to consider the case with bounded capacity. In fact, the worst case performance of a decentralized supply chain can be improved when capacity is scarce and uniform allocation is used.

THEOREM 3: With the price and profit functions defined by (1) and (2), respectively, if $K<\min \left\{z_{1} / 2, K_{2}^{+}\right\}$, then, under uniform allocation, the ratio of the supply chain profit from an equilibrium order pair over the maximum profit of the centralized supply chain is at least $1 / 2$.

PROOF: Under condition $K<K_{2}^{+}$, by Theorem 1, the allocation pair from an equilibrium order pair is $(K / 2, K / 2)$, and thus, the supply chain profit is $\pi_{S}^{L}=\left(z_{1}+z_{2}\right) K / 2-$ $K^{2}$. In the centralized supply chain, under condition $K<$ $z_{1} / 2$, the maximum profit of the centralized supply chain is obtained by allocating all capacity to retailer 1 , that is, $\pi_{S}^{*}=z_{1} K-K^{2}$. Thus, we have

$$
\frac{\pi_{S}^{L}}{\pi_{S}^{*}}=\frac{\left(z_{1}+z_{2}\right) K / 2-K^{2}}{z_{1} K-K^{2}}=\frac{z_{1}+z_{2}-2 K}{2 z_{1}-2 K} \geq \frac{z_{2}}{z_{1}} \geq \frac{1}{2},
$$

where the first inequality comes from $K<z_{1} / 2$ and the second inequality holds under Assumption 2.
IR allocation does not have a bounded worst performance ratio, even when the supplier's capacity is scarce, due to the nonexistence of an equilibrium order pair when $K<L_{1}^{+}$. Therefore, in the worst case, uniform allocation performs better than IR allocation for the supply chain.

\subsection{Computational Study}

To investigate how capacity size, allocation rule, and wholesale price affect profits of the supplier, the two retailers, and the supply chain, we conduct a computational study. Our earlier analysis normalizes the supplier's capacity cost to zero. In case there is unallocated capacity, the capacity cost has an impact on the supplier's profit. Therefore, we consider the per unit capacity cost, denoted $c$, in this section. We generate

$$
\begin{aligned}
& z_{1} \in\{0.05,0.1, \ldots, 1\}, \\
& z_{2} \in\{0.05,0.1, \ldots, 1\}, \\
& w \in\{0.05,0.1, \ldots, 0.95\}, \\
& c \in\{0.05,0.1, \ldots, 0.9\},
\end{aligned}
$$

where only combinations satisfying Assumptions 1 and 2 and $w>c$ are included. We generate capacity size $K$ in multiples of 0.01 , starting from 0.01 . Noting that uniform and IR allocations perform the same when $K>L_{1}^{+}$, we require $K \leq L_{1}^{+}$. Note that when $K<L_{1}^{+}$, no equilibrium order pair exists under IR allocation, and the expected profits are reported. Specifically, when $K \leq z_{2}-w$, we assume capacity is allocated as $(x, K-x)$, with $x$ evenly distributed between zero and $K$; when $K>z_{2}-w$, we assume capacity is allocated as $\left(x, z_{2}-w-x\right)$, with $x$ evenly distributed between zero and $z_{2}-w$. The main results of the computational study are summarized in Figs. 1-3, where the vertical axis represents the percentage of additional profit obtained under uniform allocation compared with that under IR allocation.

In Fig. 1, the horizontal axis represents the difference in the two retailers' retail prices, $z_{1}-z_{2}$. We can see that, on average, (1) uniform allocation benefits both retailers, especially

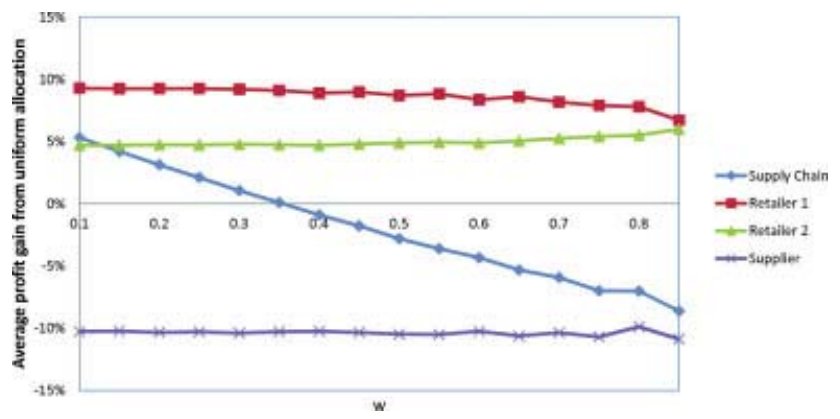

Figure 2. Profit gain as a function of $w$. [Color figure can be viewed in the online issue, which is available at wileyonlinelibrary. com.] 


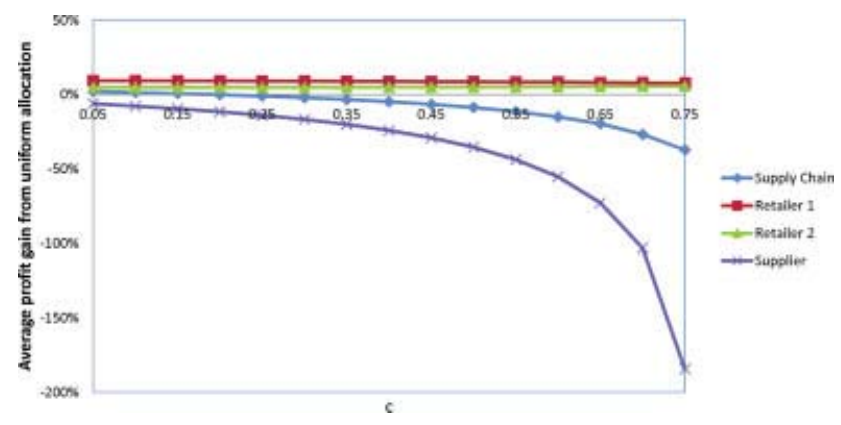

Figure 3. Profit gain as a function of $c$. [Color figure can be viewed in the online issue, which is available at wileyonlinelibrary.com.]

the high-type retailer; (2) IR allocation benefits the supplier, especially when the difference between the two retailers' retail prices is small; and (3) uniform allocation generates more profit to the supply chain when the difference between the two retailers' retail prices is not too small. In addition, except for the low-type retailer, the relative performance of uniform allocation increases with the difference between the two retailers' retail prices.

In Fig. 2, the horizontal axis represents the supplier's wholesale price $w$. The main finding is that when the wholesale price is low, uniform allocation is beneficial to the supply chain, whereas when the wholesale price is high, IR allocation generates more profit to the supply chain. For the supplier, the wholesale price has little impact on the relative performance of the two types of allocation rules.

In Fig. 3, the horizontal axis represents the supplier's unit capacity cost $c$. As the supplier's cost plays no role in the retailers' ordering decision, it has no impact on the profits of the two retailers. It is interesting to note that with increasing capacity cost of the supplier, IR allocation can be much more profitable for the supplier than uniform allocation. When the supplier's unit capacity cost is not very low, IR allocation generates more profit for the supply chain than uniform allocation.

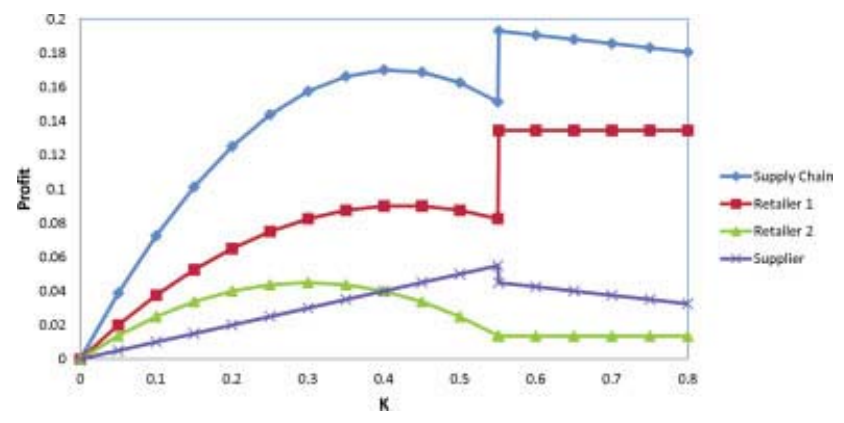

Figure 4. Instance 1: Uniform allocation. [Color figure can be viewed in the online issue, which is available at wileyonlinelibrary. com.]

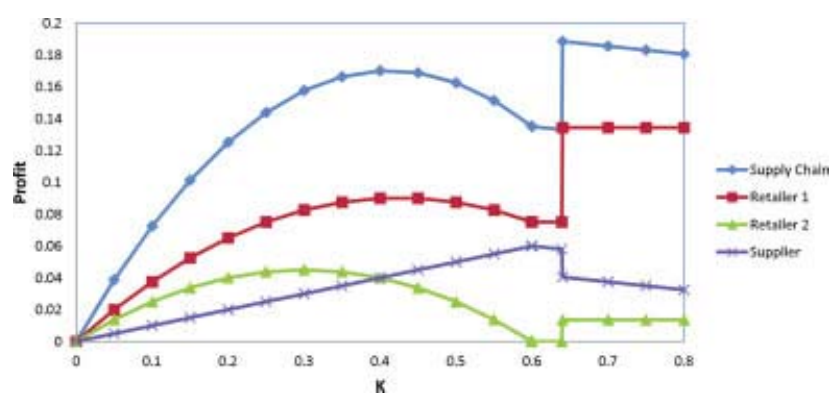

Figure 5. Instance 1: IR allocation. [Color figure can be viewed in the online issue, which is available at wileyonlinelibrary.com.]

To qualitatively illustrate the ordering and capacity allocation decisions under different capacity levels and allocation rules, we next consider two representative instances:

Instance 1. $z_{1}=1, z_{2}=0.75, w=0.15, c=0.05$, Instance 2. $z_{1}=0.95, z_{2}=0.9, w=0.15, c=0.05$,

where in Instance 1 retailer 1's retail price is significantly higher than that of retailer 2 , as quantified by $z_{1}>\left(5 z_{2}-\right.$ $w) / 4$, whereas in Instance 2 we have $z_{1}<\left(5 z_{2}-w\right) / 4$. By Theorems 1 and 2, these two instances represent the patterns of how the ordering and capacity allocation decisions change with capacity size and allocation rules. The resulting profits for different supply chain members in the two instances, under the two types of allocation rules, are depicted in Figs. 4-7.

Figures 4-7 show the profits of supply chain members for each capacity size under a capacity allocation rule. The expected profit is reported when an equilibrium order pair does not exist. Following our discussions in Section 3.3 and Figs. 4-7, we obtain the following managerial insights.

First, independent of the allocation rule used, no party in the supply chain sees its profits necessarily increasing with the supplier's capacity. This phenomenon strongly encourages the supplier to carefully control his capacity size rather

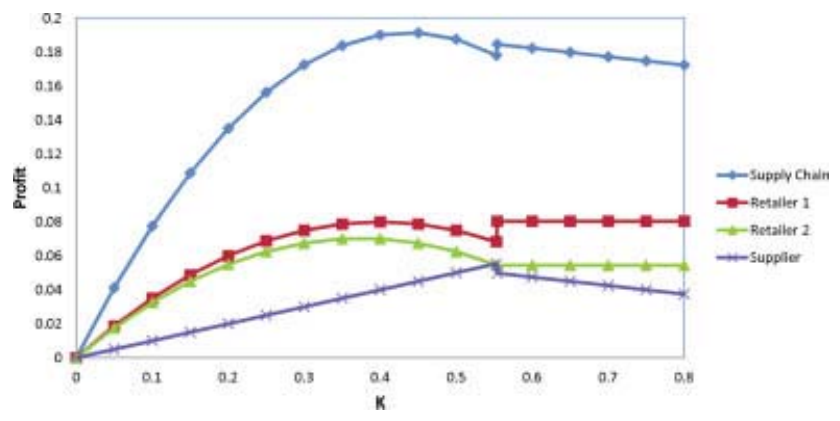

Figure 6. Instance 2: Uniform allocation. [Color figure can be viewed in the online issue, which is available at wileyonlinelibrary. com.]

Naval Research Logistics DOI 10.1002/nav 


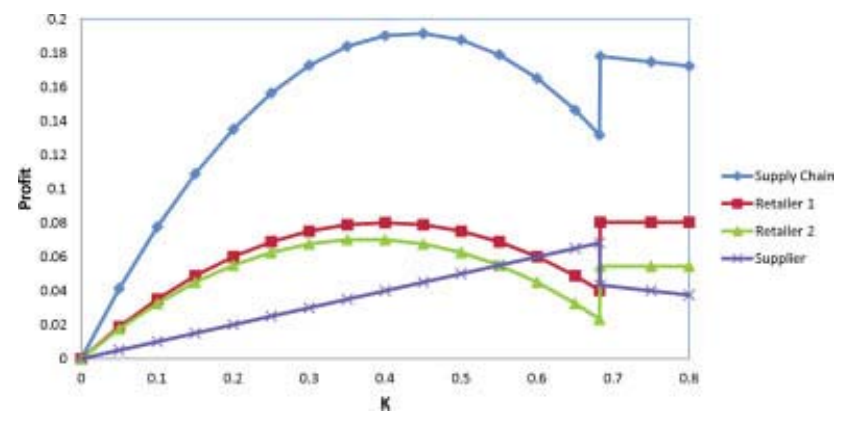

Figure 7. Instance 2: IR allocation. [Color figure can be viewed in the online issue, which is available at wileyonlinelibrary.com.]

than simply seeking a high capacity level to meet all the retailer demand. As depicted in Fig. 5, at certain capacity sizes, the low-type retailer can gain no profit and the hightype retailer can strategically order $z_{2}-w$ to drive the less competitive retailer out of the market, which, in turn, will reduce the supplier's sales capacity. In the two instances considered, the supplier's profit is maximized at a capacity size no greater than but approaching $\min \left\{L_{1}^{+}, z_{2}-w\right\}$, independent of the difference between the retail prices of the two retailers and the allocation rule used. However, this capacity size does not coordinate the supply chain, in that both retailers suffer from highly competitive ordering decisions. To better coordinate the supply chain, the supplier can reduce his capacity size to be moderately less than $\min \left\{L_{1}^{+}, z_{2}-w\right\}$ but no less than $K^{*}$.

Second, the supplier should carefully select an appropriate capacity allocation rule. The choice of allocation rule relies on supply chain configuration and the supplier's perception of his own profit and the supply chain profit. From our analysis in Section 3.3 and Figs. 1-7, IR allocation stimulates competition between retailers at their own cost to benefit the supplier, and thus, from the supplier's perspective, IR allocation outperforms uniform allocation. However, uniform allocation can better coordinate the supply chain, especially, when the difference in the two retailers' retail prices is not too small, the wholesale price is low, or the supplier's capacity cost is low. Further, under IR allocation, for a capacity size less than $L_{1}^{+}$, there does not exist an equilibrium order pair, and thus the supplier's profit is subject to variation. In addition, as is evident in Figs. 1, 4, and 5, whereas the two retailers have significantly different retail prices, different allocation rules make only a small difference in generating profit for the supplier, and thus, the supplier may choose uniform allocation without significant profit loss. However, when the two retailers have similar retail prices, IR allocation can bring more profit to the supplier at both retailers' costs. Therefore, depending on the supply chain configuration, the supplier may choose different allocation rules.

\subsection{Impact of Demand Competition}

Now, we compare capacity allocations with and without demand competition. Note that without demand competition, if the supplier's capacity is no less than the sum of the optimal sales quantities of the two retailers, then each retailer ordering her individually optimal sales quantity constitutes an equilibrium ordering decision. A question that naturally arises for capacity allocation with demand competition is whether the order pair $\left(q_{1}^{*}, q_{2}^{*}\right)$ is always in equilibrium under uniform or IR allocation when the capacity size is no less than $K^{*}$.

REMARK 3: With demand competition, under uniform or IR allocation, the order pair $\left(q_{1}^{*}, q_{2}^{*}\right)$ may not be in equilibrium when $K \geq K^{*}$.

The validity of Remark 3 is indicated by Theorems 1 and 2. The intuition behind Remark 3 is that with demand competition, when the supplier's capacity is bounded, even at a capacity size of no less than $K^{*}$, uniform or IR allocation can encourage retailers to order more than in $\left(q_{1}^{*}, q_{2}^{*}\right)$ to gain half or all the capacity, and thus more profit.

Certain properties of capacity allocation remain the same when demand competition is involved, as follows.

1. Uniform allocation guarantees equilibrium orders, whereas IR allocation does not (Theorems 1 and 2).

2. IR allocation benefits the supplier by selling more capacity, at the retailers' cost, whereas uniform allocation benefits the retailers at the supplier's cost (Remark 1, Figs. 1-3, and [4,5]).

3. Uniform allocation is most valuable to the supply chain when the wholesale price is low, whereas IR allocation is most valuable to the supply chain when the wholesale price is high (Fig. 2 and Section 6 of [5]).

Demand competition enhances the competition for scarce capacity between the two retailers and complicates the ordering and capacity allocation decisions, as follows.

1. Uniform allocation is no longer truth inducing. (Theorem 1)

2. Neither the supplier nor either one of the retailers sees its profits necessarily increasing with the supplier's capacity. In addition, the supplier can sell more with a smaller capacity size (Theorems 1 and 2).

\section{CONCLUDING REMARKS}

This work investigates the capacity allocation decisions in a supply chain in which a supplier sells a single product to two retailers at a predetermined wholesale price. The retailers 
compete for the supplier's capacity as well as for customer demand for the product. Such supply chain structures are popular in the sales of automobiles, electronics products, and textile and apparel products, among others.

We conduct an equilibrium analysis of the ordering decisions of the two retailers under two types of capacity allocation rules, and have the following interesting results. IR allocation does not guarantee equilibrium orders; uniform allocation guarantees equilibrium orders but is not necessarily truth inducing. For the same capacity size, the supplier can expect to sell at least an equal amount of capacity using IR allocation compared with using uniform allocation. Further, uniform allocation coordinates the supply chain better in the worst case and is most valuable in coordinating the supply chain when the difference between the retail prices of the two retailers is large, the wholesale price is low, or the supplier's capacity cost is low. In addition, no supply chain member sees its profits necessarily increasing with the supplier's capacity, and the supplier may sell more with a lower capacity level. Finally, capacity allocation may change the supply chain structure by driving a retailer out of the market. With these results, we provide managerial insights on the capacity and ordering decisions. Cho and Tang [7] extend this work by considering more than two retailers.

This work considers linear price functions. Other forms of price function can also be incorporated into the capacity allocation game. Consider the specific case, where

$$
p_{i}=z_{i} /\left(q_{1}+q_{2}\right), \text { for } i=1,2 .
$$

We analyze the capacity allocation game with this constant elasticity price function and find that, qualitatively, the results are similar to the case with linear price functions. Technically, this is due to the fact that with both types of price functions, the corresponding profit functions are concave with each retailer's allocated capacity. One result differs from the case with linear price functions, in that, under uniform allocation, the ratio of supply chain profit from an equilibrium order pair to the maximum profit of the centralized supply chain is no less than $1 / 2$, independent of the supplier's capacity level.

This article considers a common product sold by two retailers with different retail prices. It is helpful to consider differentiated products. In the case of linear price functions, more generally, we have

$$
\begin{aligned}
& p_{1}=z_{1}-\alpha_{1} q_{1}-\gamma q_{2}, \\
& p_{2}=z_{2}-\alpha_{2} q_{2}-\gamma q_{1},
\end{aligned}
$$

where when $z_{1}=z_{2}$, the term $\gamma^{2} /\left(\alpha_{1} \alpha_{2}\right)$ represents the degree of product differentiation, ranging from zero for independent products to one for perfect substitutes [32]. Equilibrium analysis of capacity allocation under such general price functions deserves further investigation.

\section{ACKNOWLEDGMENTS}

The author thanks the editor-in-chief, the associate editor, and the two anonymous referees for their constructive suggestions, which substantially improved the quality of this work. This research was partially supported by the summer research grant from the College of Business at the University of Michigan-Dearborn.

\section{REFERENCES}

[1] F. Bernstein and A. Federgruen, Pricing and replenishment strategies in a distribution system with competing retailers, Oper Res 51 (2003), 409-426.

[2] F. Bernstein and A. Federgruen, A general equilibrium model for industries with price and service competition, Oper Res 52 (2004), 868-886.

[3] G.P. Cachon and M.A. Lariviere, Capacity allocation using past sales: When to turn-and-earn, Manag Sci 45 (1999a), 685-703.

[4] G.P. Cachon and M.A. Lariviere, Capacity choice and allocation: Strategic behavior and supply chain performance, Manag Sci 45 (1999b), 1091-1108.

[5] G.P. Cachon and M.A. Lariviere, An equilibrium analysis of linear, proportional and uniform allocation of scarce capacity, IIE Trans 31 (1999c), 835-849.

[6] G. Cai, Channel selection and coordination in dual-channel supply chains, J Retailing 86 (2010), 22-36.

[7] S.-H. Cho and C.S. Tang, Uniform and competitive capacity allocation mechanisms with demand competition: The gaming effect. Working paper, Tepper School of Business, Carnegie Mellon University, Pittsburgh, PA, 2011.

[8] E.J. Durango-Cohen and C.A. Yano, Supplier commitment and production decisions under a forecast-commitment contract, Manag Sci 52 (2006), 54-67.

[9] F. Fang and A. Whinston, Option contracts and capacity management: Enabling price discrimination under demand uncertainty, Prod Oper Manag 16 (2007), 125-137.

[10] M.L. Fisher, What is the right supply chain for your product? Harv Bus Rev 75 (1997), 105-116.

[11] A. Ganesh, K. Laevens, and R. Steinberg, Congestion pricing and noncooperative games in communication networks, Oper Res 55 (2007), 430-438.

[12] N.G. Hall and Z. Liu, "Cooperative and noncooperative games for capacity planning and scheduling," In Z.-L. Chen, and S. Raghavan (Editors), Tutorials in operations research, INFORMS, Hanover, MD, 2008, pp. 108-129.

[13] N.G. Hall and Z. Liu, Capacity allocation and scheduling in supply chains, Oper Res 58 (2010), 1711-1725.

[14] N.G. Hall and Z. Liu, On auction protocols for decentralized scheduling, Game Econ Behav 72 (2011), 583-585.

[15] C.A. Ingene and M.E. Parry, Channel coordination when retailers compete, Market Sci 14 (1995), 360-377.

[16] C.A. Ingene and M.E. Parry, Manufacturer-optimal wholesale pricing when retailers compete, Market Lett 9 (1998), 65-77.

[17] C.A. Ingene and M.E. Parry, Is channel coordination all it is cracked up to be? J Retailing 76 (2000), 511-547.

[18] G. Iyer, Coordinating channels under price and non-price competition, Market Sci 17 (1998), 338-355.

[19] A.V. Iyer, V. Deshpande, and Z. Wu, A postponement model for demand management, Manag Sci 49 (2003), 983-1002. 
[20] S. Karabuk and S.D. Wu, Incentive schemes for semiconductor capacity allocation: A game theoretic analysis, Prod Oper Manag 14 (2005), 175-188.

[21] E. Kutanoglu and S.D. Wu, On combinatorial auction and Lagrangean relaxation for distributed resource scheduling, IIE Trans 31 (1999), 813-826.

[22] J.J. Laffont, Fundamentals of public economics, MIT Press, Cambridge, MA, 1988.

[23] H.L. Lee, V. Padmanabhan, and S.J. Whang, Information distortion in a supply chain: The bullwhip effect, Manag Sci 43 (1997), 546-558.

[24] S. Mallik and P.T. Harker, Coordinating supply chains with competition: Capacity allocation in semiconductor manufacturing, Eur J Oper Res 159 (2004), 330-347.

[25] D. Niyato and E. Hossain, A noncooperative game-theoretic framework for radio resource management in $4 \mathrm{G}$ heterogeneous wireless access networks, IEEE Trans Mobile Comput 7 (2008), 332-345.

[26] J. Raju and Z.J. Zhang, Channel coordination in the presence of a dominant retailer, Market Sci 24 (2005), 254-262.
[27] V. Padmanabhan and I.P.L. Png, Manufacturer's returns policies and retail competition, Market Sci 16 (1997), 81-94.

[28] D.M. Reeves, M.P. Wellman, J.K. MacKie-Mason, and A. Osepayshvili, Exploring bidding strategies for market-based scheduling, Decis Support Syst 39 (2005), 67-85.

[29] Y. Sprumont, The division problem with single-peaked preferences: A characterization of the uniform allocation rule, Econometrica 59 (1991), 509-519.

[30] A.A. Tsay and N. Agrawal, "Modeling conflict and coordination in multi-channel distribution systems: A review," Supply chain analysis in the eBusiness era, In: D. Simchi-Levi, D. $\mathrm{Wu}$, and M. Shen (Editors), Kluwer, Norwell, MA, 2004, pp. 557-606.

[31] M.P. Wellman, W.E. Walsh, P.R. Wurman, and J.K. MacKieMason, Auction protocols for decentralized scheduling, Games Econ Behav 35 (2001), 271-303.

[32] X. Vives, Oligopoly pricing: Old ideas and new tools, MIT Press, Cambridge, MA, 1999.

[33] T. Xiao and X. Qi, Price competition, cost and demand disruptions and coordination of a supply chain with one manufacturer and two competing retailers, Omega 36 (2008), 741-753. 